\title{
Put my name on that paper: Reflections on the ethics of authorship
}

Martin McKneally, MD

From the Department of Surgery and Joint Centre for Bioethics, University of Toronto, Toronto, Ontario, Canada.

Received for publication Aug 24, 2005; accepted for publication Sept 27, 2005.

Address for reprints: Martin McKneally, MD, 77 Forest Grove Dr, Toronto, Ontario M2K 1Z4, Canada (E-mail: martin.mckneally@ utoronto.ca; dmckneally@ sympatico.ca).

J Thorac Cardiovasc Surg 2006;131:517-9

$0022-5223 / \$ 32.00$

Copyright (C) 2006 by The American Association for Thoracic Surgery

doi:10.1016/j.jtcvs.2005.09.060
A t a recent meeting of the Journal's Editors, questions arose about how many authors can reasonably be listed on a published paper, and what qualifies a contributor to be an author. Editor Andy Wechsler asked me to explore some of these issues, including their ethical implications.

Authorship certainly involves ethical values, such as truthfulness, trustworthiness, and fairness. Safeguarding the integrity of the Journal as a trusted official publication of our profession is an ethical obligation of the editors, peer reviewers, authors, and readers. I will try to sketch a conceptual framework for thinking about authorship that includes some definitions, ethical considerations, and authorship policies that have been adopted by other journals. Then I'll make some suggestions for readers' consideration and comments.

\section{Conceptual Framework \\ Defining Authorship}

An author is an originator, based on the word's Latin and Greek roots, and authorship is a justified claim to be an originator or progenitor. As the concept of authorship in medical science has evolved, authorship has come to include a claim to originality or other scientific value of published work, responsibility for the veracity and reliability of the report, and ownership of the work as intellectual property. Though copyright may be transferred, for example, to The American Association for Thoracic Surgery as a condition of publication in the Journal, the author retains scientific and public responsibility and credit for the invention, discovery, or formulation reported.

The defining qualifications for a valid claim to authorship have been the subject of helpful discussion by a number of thoughtful editors and writers. Because authorship is a somewhat notional concept (there are many notions of what it means to be an author and no widely accepted scientific definition), opinions vary about minimum qualifying standards. Drummond Rennie, the deputy editor of the Journal of the American Medical Association, has been a champion of explicit clarification and validation of authorship claims. His article, "When Authorship Fails: A Proposal to Make Authors Accountable," described a new and quite rigorous definition that has been adopted by several journals and endorsed by the International Committee of Medical Journal Editors (ICMJE). The essential authorship criteria of the ICMJE are:

“1) substantial contributions to conception and design, or acquisition of data, or analysis and interpretation of data; 2) drafting the article or revising it critically for important intellectual content; and 3) final approval of the version to be published. Authors should meet conditions 1, 2, and 3...

Acquisition of funding, collection of data, or general supervision of the research group, alone, does not justify authorship."2 Many of our authors would not meet these standards, and we are unlikely to adopt them in this form, but what criteria should we apply? Let's begin with ethics, often a useful source of guidance.

\section{Ethical Considerations}

Truthfulness of authors' claims is a foundational ethical value in our framework. Truthfulness has long been thought to be reasonably assured by the well developed standards of the scientific method, critical peer review by members of the editorial 
board and expert guest reviewers, scrutiny by readers and practitioners, and the requirement for confirmatory studies by other investigators. False claims, including unjustified claims to authorship, are violations of the foundational value of truthfulness. Inaccuracies are ethically distinguishable from false claims. There is a moderately high rate of error or exaggeration of benefit in even the largest and widely cited clinical trials, as evidenced by contradiction $(16 \%)$ or correction of exaggerated claims $(16 \%)$ in subsequent large series. ${ }^{3}$ These are not deliberate attempts to deceive; they are generally errors in the best sense of the word-originally derived from "wandering from the path." Sometimes the path to truth is unknown, and sometimes the statistical power of the study is insufficient to detect it. Some have inferred from the error rate in published studies that the process of anonymous voluntary peer review may be flawed. ${ }^{4}$ I believe it is an admirable example of responsible democratic decision making by recognized experts who have merited the trust of their peers. Their fallibility is part of the essence of the scientific process, which will continue to make progress through trial, error, and correction. Peer reviewers serve a critically responsible role. Their hard work greatly enhances many of the manuscripts received by the journal. ${ }^{5}$ For more detailed discussion of peer review, see http://www.ama-assn.org/public/peer/peerhome.htm, the Web site of the International Congress on Peer Review and Biomedical Publication.

Trustworthiness is a more complicated component of our conceptual framework. It builds on truthfulness, but includes the richly nuanced requirement for fidelity to trust. Trustworthy authors (and, by extension, reviewers and editors) not only publish what they believe to be true, they also strive to minimize bias in selection, rejection, and interpretation of the truthful information they present to trusting readers. A troubling threat to trustworthiness of scientific publication arises when biased judgments are made about reporting or publishing findings that conflict with the interests of sponsoring organizations, institutions, advertisers, reviewers, editors, or governments. While premature publication of problems with a device or drug may precipitate an unreasonable devaluation of a company's market position, delay in publication of unfavorable findings to protect market share harms patient subjects and erodes public trust in industrial partners and research scientists.

Fairness, the public face of justice, requires that public credit be awarded to originators for original work. Fairness becomes less discernable when multiple authors contribute to a scientific report. Like a major surgical operation, most contemporary scientific studies in our field are team efforts that require working together synergistically toward a goal that none of the contributors could achieve alone. Our operative reports, by convention, clearly define some of the roles of participants. We could adopt a new convention for our journal reports that is similarly clear and transparent in describing various roles of the "authors" who contribute to our scientific studies. In the ideal, these roles and responsibilities, including status and order of authors, would be assigned and agreed upon in advance. In reality, levels of participation and responsibilities often change over time. This understandable fluidity in the scientific process can lead to assumptions that are not mutually shared or claims to authorial credit that are not mutually accepted.

In the majority of cases, decisions within the group of contributors about the distribution of credit are primarily a matter of policy and courtesy, rather than ethics, but ethical principles can be applied to help authors reason through the issue of assignment of credit. For example, the principle of justice requires that recognition of our colleagues should be proportionate to the value of their contribution. The complexity and lack of transparency in the evolution of authorship policy decisions makes objective evaluation of claims and counter-claims about fairness impracticable. There are no established guidelines or reference points for making reliable judgments about the fairness of these decisions. The enigmatic implications of the order of authors are unique to scientific publication. In the absence of explanatory description, the meaning of the order of authors is encrypted in code that is variously deciphered by readers, using a vague and unratified convention. The first author is generally assumed to be a principal author, and the last author position is usually decoded as the senior author, although the meaning of these terms is unspecified. Additional internuncial contributors are assumed to be listed in descending order of the importance of their contribution. These interpretations and the responsibilities they imply are uncertain at best.

\section{Some Practical Suggestions}

Here are a few distinctions that would help me as a reader to understand what went on behind the scenes. Perhaps we could include them in the bylines on the title page, where we identify the authors' affiliations.

Principal authors take direct responsibility for the manuscript. In addition, they often direct or manage the conduct of the research project, and serve as guarantors of the integrity of the study. Principal authors fulfill the three ICMJE criteria of authorship described above.

Contributors enable the completion of the scientific mission and publication of the results in a rich variety of ways. Some may not fully qualify as authors despite their valued contributions. A useful explanatory taxonomy of contributions is evolving in several journals, such as the Journal of the American College of Surgeons, using terms such as: proposed the original idea, co-developed the project, coordinated the project, provided and cared for study patients, acquired the data, wrote numerous drafts, produced the final draft, contributed expertise on ethics/statistics/etc. 
A concise, descriptive, narrative approach is used in the Canadian Medical Association Journal. In only one column inch of space at the end of the report, the CMAJ is able to inform readers that:

[Dr. Author] was the primary author and was responsible for the design of the study and the analysis and interpretation of the data. [Dr. Data] supervised the development of the database, provided input on the study design and reviewed and approved the final manuscript. [Dr. Investigator] was the principal investigator of the study, supervised the acquisition and interpretation of the data, and provided critical input to the manuscript.

When described clearly, contributions can be used to assign academic credit more precisely. The requirement to specify participants' contributions to the project may also reduce claims for the status of author to a realistic number.

Senior authors generally direct, oversee, and guarantee the authenticity of the work reported. Senior authors could be more explicitly identified and their roles specified. They often determine policy for publication, for example, choice of venue for presentation, designation of the team member who should present the oral version of the study, who should write the first draft, who should be listed as authors, and the order of names on the list of authors. Senior authors implicitly take responsibility for the work's scientific accuracy, valid methodology, analysis, and conclusions.

Gift authors are often department or service chiefs who appear on the title page of a report without necessarily contributing substantially to the work. Explicit acknowledgment of their role would help eliminate this sometimes unfair and occasionally dangerous custom. A particularly troublesome recent variant of gift authorship is the entre- preneurial practice of preparing scientific reports on a device or pharmaceutical agent, carefully ghost-written by an employee of the manufacturer to match a prominent researcher's style. The report, ready to send to a high impact journal, is presented to the proposed gift author, sometimes accompanied by a substantial honorarium!

\section{Closing Comments}

The JTCVS receives submissions from a wide variety of venues around the world, and we have not insisted on a rigid or formal definition of the contribution of each author. We encourage thoughtful reflection on the roles and responsibilities of authors, and we will continue to foster a spirit of fairness and transparency as we develop our policies and guidelines. Your Editors look forward to correspondence from our readers, to "contribute, co-develop, co-refine, and clarify" the richly ambiguous and important issue of authorship for our Journal.

I am grateful to Andy Wechsler and Gene Blackstone for helpful comments on earlier versions of this editorial.

\section{References}

1. Rennie D, Yank V, Emanuel L. When authorship fails: a proposal to make contributors accountable. JAMA. 1997;278:579-85.

2. International Committee of Medical Journal Editors. Uniform Requirements for Manuscripts Submitted to Biomedical Journals: Writing and Editing for Biomedical Publication, updated October 2004. http://www. icmje.org/ (accessed August 22, 2005).

3. Ioannidis JPA. Contradicted and initially stronger effects in highly cited clinical research. JAMA. 2005;294:218-28.

4. Kranish M. Flaws are found in validating medical studies; many see need to overhaul standards for peer review. The Boston Globe 2005 Aug 15.

5. Wechsler AS. Peer review: recognition and responsibility. $J$ Thorac Cardiovasc Surg. 2001;122:419-20. 